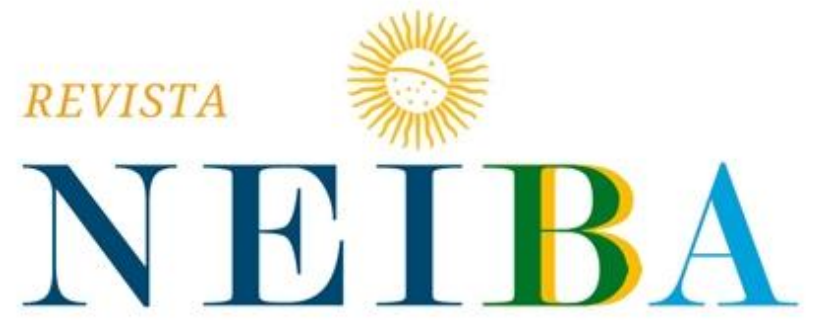

CADERNOS ARGENTINA-BRASIL
Volume 8, 2019, p. 01-17

DOI: 10.12957/neiba.2019.39455 | e39455 | ISSN: 2317-3459

\title{
CALLE TOMADA. LA PARADOJA DE LA INDIGNACIÓN EN BRASIL Y LA DISPUTA POR LA SOCIEDAD
}

STREET TAKEN. THE PARADOX OF OUTRAGE IN BRAZIL AND THE DISPUTE OVER SOCIETY

Natalia Razovich ${ }^{1}$

${ }^{1}$ Universidad Nacional de Rosario (UNR), Rosario, Santa Fe, Argentina. E-mail: natirazovich@gmail.com. ORCID: 0000-0001-5670-7863.

Recebido em 15 jan. 2019 | Aceito em 03 dez. 2019. 


\section{RESUMEN}

En un contexto mundial de proliferación de revueltas y manifestaciones, desde junio de 2013 las calles de Brasil han sido testigo de su propio movimiento de indignados. Abriendo un proceso de crisis social y político institucional, un sinnúmero de indignaciones fueron llevadas a escena por actores y grupos dispares que dirimieron virtual, territorial y simbólicamente la disputa por la sociedad. En este marco, el objetivo de este trabajo es centrarse en la reflexión y análisis del ciclo de protesta social abierto en junio de 2013 hasta la concreción del impeachment a Dilma Rousseff en 2016.

Palabras Claves: Protesta; Movimientos Sociales; Indignación.

\section{ABSTRACT}

In a global context of proliferation of revolts and manifestations, the streets of Brazil have witnessed their own outrage movement since june 2013. Opening a social, political and institutional process of crisis, several outrageous were taking to scene by different groups and actors, which settle virtual, territorial and symbolically the dispute for society. In this context, the aim of this article is to focus on the reflexion and the analysis of the social protest cycle opened in june 2013 until Dilma Rousseff impeachment concretion in 2016.

Keywords: Protest; Social Movements; Outrage.

Antes de alejarnos tuve lástima, cerré bien la puerta de entrada y tiré la llave a la alcantarilla. No fuese que algún pobre diablo se le ocurriera robar y se metiera en la casa, a esa hora y con la casa tomada.

Julio Cortázar. Casa tomada.

\section{INTRODUCCIÓN}

A partir de junio de 2013, la protesta social en Brasil se convirtió en un elemento ineludible del reciente ciclo político. Grupos nuevos y demandas dispares, se han enfrentado en las calles en torno a un proyecto de sociedad. El objetivo de este artículo es analizar el ciclo de protesta social en Brasil desde junio de 2013 hasta la concreción del impeachment a Dilma Rousseff, reflexionando acerca de la presencia y la figura de movimientos sociales detrás del mismo 
Mientras a nivel mundial se habla de revueltas de reivindicación popular e indignaciones que parecen desestimar de todo tipo de organización colectiva y elevar la individualización ciudadana, se abre el interrogante acerca de las categorías analíticas protesta social y movimiento social. ¿Tienen, las mismas, necesariamente una relación directa? más específicamente, cabe preguntarse si ¿toda protesta social cuenta con algún movimiento social previo detrás? y si ¿toda protesta constituye o da forma a un movimiento? Actualmente, en el nuevo contexto expansivo de manifestaciones al que se asiste desde el 2011, ¿qué implica un movimiento social? Por lo general, mediáticamente los términos protesta social y movimiento social son empleados como sinónimos. Se intentará demostrar que, en la actualidad es cada vez más difícil percibir tales categorías de forma excluyente, por ello resulta sumamente útil pensarlas como un binomio, como una suerte de comunión o combinación de las dos perspectivas sociológicas tradicionales abocadas al estudio de la acción colectiva.

En primer lugar, se abordará un breve recorrido conceptual de la acción colectiva, haciendo hincapié en la propia historia y perspectiva latinoamericana para su abordaje. En tal cometido, se tomará la distinción propuesta por Maristella Svampa (2017) entre movimientos en sentido fuerte y en sentido débil como forma de entender a las protestas y a los movimientos de modo simbiótico. Se continuará apuntando, en base a los aportes sociológicos de Manuel Castells (2012) y Boaventura De Sousa Santos (2015), una breve descripción de la modalidad que adopta el término de movimientos sociales en el actual contexto global de indignaciones, dando cuenta que la nueva forma espacial de los movimientos sociales permite contemplarlos tanto en su sentido como débil como fuerte.

En segundo lugar, se tratará de discernir si existe una línea de continuidad en el ciclo de protesta que irrumpe en Brasil, en cuanto a la presencia de actores, reclamos y demandas. Se dará cuenta de que el mismo no fue un proceso lineal y su deriva resultó en una paradoja intrínseca entre dos momentos diferenciados, referentes a los reclamos por más Estado y más mercado, a la que en este trabajo se hace referencia como "paradoja de la indignación". Se propone como punto de inflexión entre tales momentos la reelección en 2014 de Dilma Rousseff, dando cuenta, a partir del mismo, de un cambio en la correlación de fuerzas mayoritarias en las calles y de una creciente polarización social. Las calles, metafóricamente, representaban a la sociedad en disputa.

\section{DE MOVIMIENTOS Y PROTESTAS, LAS VARIANTES DE LA ACCIÓN COLECTIVA}

Enfoques de la acción colectiva y tradición latinoamericana: 
Hasta la década del sesenta, la acción colectiva era comprendida desde la teoría marxista de luchas de clases y los movimientos sociales hacían referencia exclusivamente al movimiento obrero. Sin embargo, en el marco de los Estado de Bienestar adviene un ciclo de acción colectiva que no encuadraba en tales parámetros ya que el descontento social no podía ser explicado en base a las cuestiones materiales de existencia.

Con el mayo francés, como parte aguas histórico, se constituyen desde la sociología dos perspectivas teóricas para abordar la acción colectiva, la de movilización de recursos, desde la escuela norteamericana y la de los nuevos movimientos sociales, desde la escuela europea. Mientras el primer enfoque responde a una lógica instrumental con el objetivo de dilucidar el cómo de las manifestaciones, el segundo responde a una lógica expresiva preocupada por el porqué de las mismas. Tales perspectivas refieren a modelizaciones y estilizaciones teóricas que tienden a especificar un tipo específico y diferenciado de acción colectiva, pero ello no quita el hecho de que en las movilizaciones sociales siempre se encuentren presentes ambas lógicas, aunque por lo general una prevalezca sobre la otra (Iglesias, 2008).

Tal como explica Esteban Iglesias (2008), el primer enfoque, cuyos mayores exponentes son Sidney Tarrow (2004) y Charles Tilly (2000), prioriza la organización y la acción contenciosa como momento político de la acción colectiva. Tarrow plantea su abordaje en términos de "estructura de oportunidades políticas", según la cual, se presentan determinadas circunstancias particulares en las que la acción colectiva tiene lugar, incluso, sucede que "la lógica cada vez más amplia de la acción colectiva conduce a resultados en la esfera política en la que los movimientos que iniciaron el ciclo pueden acabar teniendo cada vez menor influencia" (Tarrow 2004, p. 52). Por su parte, la teorización de los "repertorios de contención" realizada por Tilly, da cuenta de la variación del modo que puede asumir la acción colectiva a largo plazo. En términos del propio autor, "el término repertorio captura la combinación de elaboración de libretos históricos e improvisación que caracteriza generalmente a la acción colectiva" (Tilly 2000, p. 14).

Por su parte, la segunda perspectiva, que tiene a Alain Touraine (1998) como máxima expresión, prioriza cuestiones culturales y concierne a la cuestión identitaria y la conformación de sujetos colectivos. Para este autor, un movimiento social se define en torno a tres principios: el de identidad, el de oposición y el de totalidad, siendo el de totalidad el que reviste un carácter definitorio, al referir a la elaboración de un proyecto mediante el cual se pondría en cuestión la historicidad de la sociedad (Iglesias 2012, p. 116). De este modo, "un movimiento social (...) siempre quiso abolir una relación de 
dominación, hacer triunfar un principio de igualdad, crear una nueva sociedad" (Touraine 1998, p. 102).

Svampa (2017, p. 30) menciona que en América Latina la teoría de la acción colectiva pasó por la influencia de ambos paradigmas mediante la priorización del concepto de movimientos sociales o de protesta. En los años 90 la influencia del neoliberalismo en todos los órdenes de la vida dio lugar a repertorios de acción tanto tradicionales, marchas y manifestaciones, como nuevos, saqueos, puebladas y piquetes, que inicialmente se abordaron priorizando la noción de protesta vinculada al abordaje de movilización de recursos. Sin embargo, desde el levantamiento de Chiapas en 1994 y a partir de los albores del siglo XXI, se daría lugar a un nuevo ciclo de acción colectiva que rehabilitó nuevamente el protagonismo del concepto de movimientos sociales a partir de la cuestión identitaria, en torno a una acumulación de luchas a nivel mundial en contra de las reformas neoliberales.

En el mismo sentido, Seone, Taddei y Algranati (2006, p. 230) destacan que no sólo se trataba del inicio de un nuevo ciclo de protestas sociales, sino también de que el mismo apareció encarnado en sujetos colectivos con características particulares y diferentes de aquellos que habían ocupado la escena pública en el pasado. A su vez, señalan que ello acontecía de manera casi simultánea al crecimiento del conflicto en otras regiones del planeta, en un proceso que signará la constitución de un espacio de convergencia internacional en oposición a la mundialización neoliberal al que se ha denominado movimiento "antiglobalización" o "altermundialista". Respecto a tal convergencia mundial de inconformidades, resistencias y rebeldías, Ana Esther Ceceña (2002, p. 12) realiza una distinción esquemática entre movimientos del norte y movimientos del sur. Mientras el posicionamiento frente al neoliberalismo de los movimientos del norte implicaba el reclamo de sistemas de regulación frente a la globalización y sus instituciones, el sur denunciaba las premisas fundacionales de la sociedad capitalista.

Así, la producción latinoamericana daba cuenta del surgimiento de nuevos movimientos de base territorial tanto en el mundo rural como también en el espacio urbano, constituidos, por lo general, en relación a su identidad étnico-cultural - los movimientos

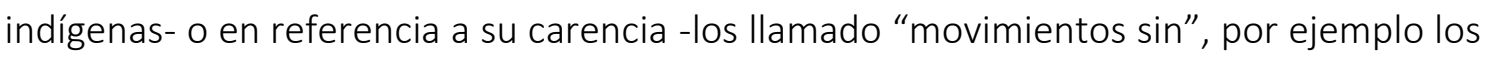
sin tierra, sin techo o sin trabajo- (Seone, Taddei y Algranati 2006, pp. 232-233).

Según Raúl Zibechi (2003, p. 185), los nuevos movimientos sociales más significativos (Sin Tierra y seringueiros en Brasil, indígenas ecuatorianos, neozapatistas, guerreros del agua y cocaleros bolivianos y desocupados argentinos) poseían, al margen de sus diferencias espaciales y temporales, una serie de rasgos comunes, como ser: su carácter antineoliberal, la territorialización, la afirmación de una identidad propia y autónoma 
del Estado y la sustitución de prácticas instrumentales de luchas como la huelga por técnicas autoafirmativas. Sin embargo, el escenario actual difiere del de finales de los noventa y comienzo del siglo XXI y a la luz de los acontecimientos dicha caracterización resulta anacrónica para comprender la oleada de protesta social en Brasil desde el 2013 y las primaveras alrededor del mundo.

Una nueva convergencia mundial de manifestaciones, que Bringel y Pleyers (2015) han denominado "geopolítica de la indignación global", obliga a repensar las categorías analíticas o bien flexibilizarlas. Es por ello que este trabajo rescata la distinción que realiza Svampa (2017) en el uso analítico de la noción misma de movimiento social, ya que se considera que con la misma tiene lugar una suerte de superación a la dicotomía excluyente entre las categorías de protesta social y movimiento social, permitiendo pensarlas como un binomio, como una comunión de los tipos de acción colectiva arriba referidos.

La autora citada refiere, por un lado, a los movimientos sociales en sentido fuerte, aludiendo a la idea de un actor o movimiento social que cuestiona la lógica de dominación, cuya acción es irreductible a la institucionalización e implica la posibilidad de pensar los movimientos sociales como sujetos potencialmente antagónicos y emancipatorios. Por otro lado, el sentido débil de la noción de movimientos sociales, alude a un tipo de acción colectiva que intencionalmente busca modificar el sistema social establecido, o defender algún interés material; una acción contenciosa e intencional que da cuenta de una continuidad organizativa (Svampa 2017, p. 33).

Se considera que tal enfoque resulta pertinente para la comprensión de un contexto global de efervescencia social que revaloriza la protesta y la ocupación de espacios públicos, con la característica de que los individuos que se movilizan, en su gran mayoría, lo hacen por fuera de toda institución u organización y ello ha recibido la denominación, principalmente mediática, de movimientos de indignados. En definitiva, con el presente recorrido teórico, se procura flexibilizar el sentido fuerte del término movimientos social y revalorizar su sentido de débil, es decir, el momento contencioso.

\section{Indignaciones mundiales}

Las movilizaciones callejeras en Brasil desde junio de 2013, englobadas junto a las movilizaciones ciudadanas de la Primavera Árabe y de los indignados del sur europeo (Grecia, España, Portugal e Italia), al movimiento estudiantil chileno, al movimiento Occupy WallStreet en Estados Unidos y al movimiento \#Yosoy132 contra el fraude electoral en México, forman parte de una "ola de reivindicación popular" de nuevo tipo que tiene lugar desde el 2011 en todo el mundo (De Sousa Santos 2015, p. 19). 
Manuel Castells (2012, p. 213), se refiere a tales fenómenos como "redes de indignación" que conforman los movimientos sociales en red, propios de nuestro tiempo, los cuales adoptan nuevas modalidades y significaciones a partir de la utilización de las nuevas tecnologías de información. La nueva forma espacial de los movimientos sociales en red estaría constituida por un espacio de autonomía generado a partir del híbrido entre el ciberespacio y el espacio urbano. La difusión de Internet y una variedad de herramientas de software social han permitido establecer redes horizontales de comunicación interactiva, generando una autocomunicación local y global que es capaz de convertirse en un movimiento cuando los espacios urbanos son ocupados físicamente.

Si bien cada movimiento responde a las características propias de cada país, el común denominador es la indignación ciudadana por lo que en tales revueltas prevalece la negatividad sobre la positividad. Los autores citados concuerdan en que estos nuevos movimientos surgen de una crisis de legitimidad de las instituciones al haber sido cooptadas o colonizadas por el mercado y de una democracia representativa liberal derrotada como consecuencia de la crisis financiera del 2008. Por tal motivo, discurren por fuera de las organizaciones tradicionales, siendo las calles los únicos espacios públicos libres.

Al respecto, Pierre Rosanvallon (2015, p. 183) refiere al advenimiento de una democracia negativa marcada por un clima de desilusión en la cual el ciudadano negativo no es un ciudadano pasivo sino que afirma su participación en la vida pública pero en forma hostil, es decir en favor de un rechazo. La democracia negativa se establece en una suerte de democracia directa regresiva. Desde una perspectiva más positiva, Pablo Vommaro $(2015$, p. 54$)$ destaca que tales procesos de movilización social encuentran en los jóvenes sus principales impulsores, dando lugar a una dinamización del conflicto social y a la conformación de la agenda pública.

En conclusión, se destaca que las indignaciones y ocupaciones no fueron obra de los movimientos tradicionales sino del sujeto común, personas que se consideran despolitizadas, ciudadanos sin experiencia participativa que se movilizan en base una memoria acerca de un estado social de derechos y en reclamo de una democracia real (De Sousa Santos 2015, p. 27). La mayoría de los movimientos de indignados en sus inicios son espontáneos y requieren de una movilización emocional, desencadenada por alguna chispa de indignación y por la esperanza de la posibilidad de un cambio como resultado de los ejemplos de levantamiento que han tenido éxito en otras partes del mundo. De este modo, mediante la transmisión en red de imágenes y mensajes a través de internet, cada revuelta inspiraría la siguiente, propagándose por efecto contagio (Castells 2012, p. 20). 
Por su parte, Breno Bringel (2013) remarca que si bien es importante situar las protestas en Brasil en un contexto más amplio de indignación global en el que se comparten símbolos y demandas, las indignaciones no son un movimiento sino más bien, un estado de ánimo. Según el autor, la indignación, la molestia, la ira o el odio aún no se cristalizan en torno a una acción política estructurada y la pregunta principal, tanto para en Brasil, como para otros países que han experimentado movimientos indignados, es cómo canalizar el sentimiento y convertirlo en un movimiento social.

\section{LA PARADOJA DE LA INDIGNACIÓN}

El ciclo de protesta social abierto en el año 2013 no se constituyó en un proceso lineal y homogéneo, sino diferenciado en torno a reclamos y actores. Su deriva condujo al proceso de crisis político institucional representado en el impeachment a Dilma Rousseff en 2016. En términos de Tarrow (2004), puede sostenerse que los sucesos de junio abrieron una estructura de oportunidades para nuevos grupos que aprovecharon un ambiente de crispación social propicio para movilizar sus recursos y alzar sus demandas. La gente participa en acciones colectivas "como respuesta a un cambio en la pauta de las oportunidades y restricciones políticas y, mediante el uso estratégico de la acción colectiva, genera nuevas oportunidades que serán aprovechadas por otros en ciclos de protesta cada vez mayores" (Tarrow 2004:45).

En consonancia con la investigación de Dolores Rocca Rivarola (2016), este trabajo, plantea la existencia de dos momentos diferenciados de la protesta social. La autora citada hace referencia a los aspectos contrastantes de los contextos de protestas, centrándose en junio de 2013 y el Fora Dilma que inició en 2015. El presente artículo incorpora la idea de que tal distinción se corresponde con la entrada en escena de nuevos grupos, que se disputaron el control de las calles y pondrían en juego el sentido de un modelo de sociedad.

En dicha línea de análisis, con meros fines explicativos, se propone como punto de inflexión entre estos dos momentos la reelección de Dilma Rousseff en 2014. Sin embargo, tal punto es arbitrario y se utiliza a modo de clarificación y esquematización ya que se reconoce que tales momentos no son herméticos sino que se dan, incluso, de modo simultáneo. Tal esquematización puede visualizarse en las Jornadas de Junio de 2013, donde se hace presente el Movimiento Passe Livre (MPL), y en la Marcha de Marzo de 2016, donde se encuentran ya afianzados el Movimiento Brasil Livre (MBL), Revoltados On Line y Vem pra Rua (VPR). A partir del antes y el después de la reelección, es posible dilucidar un cambio en el peso relativo de unos y otros actores, que terminará por dar cuenta de un fenómeno poco común, la derecha en todos sus 
espectros comenzaba a tomar y ganar la calle y el sentido de una protesta que un principio no le fue propia (Domingues 2016, p. 67).

Según las encuestas de IBOPE, el $89 \%$ de los manifestantes no se sentía representado por los partidos políticos, un $46 \%$ afirmaba que era la primera vez que se manifestaba en las calles y un $62 \%$ supo de la convocatoria por Facebook y otras redes sociales. En rasgos generales, Brasil comenzaba a tener su propio movimiento de ciudadanos indignados, pero el mismo no se constituiría en un proceso lineal. Mientras en un primer momento, las indignaciones en movimiento tenían detrás de sí un movimiento en sentido fuerte que las vehiculizaba, en el segundo momento de la protesta, se encuentran elementos de movimientos sociales en sentido débil, dado que lo más trascendental era el choque, el número, y la demostración contenciosa en torno a un reclamo que cada vez fue más concreto: el impeachment a Dilma Rousseff.

Los sucesos de Junio de 2013

A comienzos del año 2013, según una encuesta realizada por IBOPE, Dilma Rousseff contaba con su mayor porcentaje de aprobación, un 63\% de la población consideraba bueno su gobierno. Para el mes de julio, dicha cifra se redujo drásticamente a la mitad, contando con sólo un 31\%.

Si bien, en el 2013 Brasil no se encontraba en una situación recesiva y los niveles de empleo se mantenían estables, la economía estaba estancada y la inflación crecía al $6 \%$. Como consecuencia de la baja en los precios de las commodities, a partir de 2011 comenzaría una etapa de desaceleración de la economía y agotamiento de la agenda de avances sociales (Anderson 2016, p. 38).

Las jornadas comenzaron el 6 de junio de 2013, con la convocatoria del MPL a una nueva manifestación en San Pablo en contra del aumento del 20\% del transporte público. Este movimiento, conformado por jóvenes, universitarios y profesionales, en torno a la reivindicación por el "pasaje gratuito estudiantil", se constituyó como organización federal en el año 2005. Definiéndose como un movimiento autónomo, independiente y apartidario, desde ese entonces, su objetivo pasó a hacer el "pasaje gratuito para toda la sociedad" utilizando repertorios de protesta. Posteriormente, a partir del contacto con los sectores populares de las periferias urbanas, familias pobres y negras de que sufrían el desalojo arbitrario consecuente de la reformulación de la ciudad para albergar las copas de fútbol, adoptaron el término "tarifa cero", como resultado de entender el derecho al transporte de una manera amplia, como un derecho a la ciudad (Zibechi 2013, p. 22). 
Las jornadas de movilización organizadas por el MPL solían contar con alrededor de 2000 o 3000 personas que salían a las calles y eran rápidamente reprimidas por la policía (Breda 2016, p. 6). Sin embargo, para mediados de junio, a las convocatorias se sumarían otros movimientos y organizaciones sociales hasta desbordar a la población en general. Las dimensiones de la protesta trascendieron al movimiento organizador y se convirtieron en netamente ciudadanas, dando lugar en Brasil a su propio movimiento de indignados (Castells, 2013). La fuerte represión policial contribuía a encender la chispa para salir a las calles, sin embargo, el ingreso de nuevos actores como el grupo anarquista Black Blocs, iría generando una merma en el apoyo social (Goldstein 2019, p. 50).

A medida que las manifestaciones se multiplicaban en las mayores ciudades de Brasil, las demandas excedieron el reclamo en contra del aumento del precio de boleto hacia el costo de calidad de vida en general. Mejoras en los servicios públicos, la educación y la salud fueron los principales gritos de indignación. En el marco de la Copa de Confederaciones, se exigían patrones FIFA para los servicios y bienes públicos, frente a la percepción de que las inversiones deportivas no generaban impacto alguno en la vida cotidiana.

Los anteriores años de bonanza impulsaron una revolución en las expectativas de movilidad social ascendente (Goldstein 2019, p. 49). Las manifestaciones pusieron en juego tanto el sentido y la producción de lo público, como los usos de los dineros estatales, las connivencias con la empresa privada, el uso y apropiación del espacio urbano y las formas de participación política (Vommaro 2015, p. 62). En este sentido, son enmarcadas en las limitaciones del modelo de acumulación y de los avances políticos y sociales de los gobiernos progresistas. Más allá de los indicadores favorables de desarrollo social, no se logró llevar a cabo una verdadera política de redistribución del ingreso sino que más bien se estimuló el consumo, mientras se desatendieron los bienes públicos (Anderson 2016, p. 40).

En esta línea, también se orientan los análisis que no reconocen la presencia de una nueva clase media a partir del aumento de la renta dado que, tal indicador, invisibilizaría la persistencia e incluso profundización de las desigualdades sociales. Jessé de Souza prefiere dar lugar al término de una nueva clase trabajadora (citado por Tible 2013, pp. 8-9) mientras que, Ruy Braga (2013) hace mención al precariado, para referir a una masa trabajadora descalificada o semicalificada, que entra y sale rápidamente del mundo del trabajo y está insatisfecha con el modelo de desarrollo. Por su parte, André Singer (2013, p. 27) sugiere la hipótesis de que junio de 2013 fue simultáneamente, obra de las expresiones de inconformidad tanto de una clase media tradicional como de, lo que prefiere denominar, un nuevo proletariado, aludiendo a jóvenes con baja 
remuneración y malas condiciones laborales cansada por el mal funcionamiento y provisión de los bienes y servicios públicos.

En este primer momento de la protesta, es posible afirmar que se asistió a una demanda por más y un mejor Estado por parte de una clase media y media baja que impugnaba la merma en su acceso a derechos básicos mientras las inversiones se dirigían a establecer un ambiente propicio para el turismo de cara a los megaeventos deportivos, objetivos no prioritarios de lo que supone un gobierno progresista (Goldstein y Rezende 2016, p. 8). Las indignaciones eran por el derecho a una ciudadanía plena, frente al déficit de los servicios públicos, el costo de la vida y el saqueo urbano, inmobiliario y especulativo que acompañaba la disminución de la capacidad adquisitiva y la reducción de gastos sociales. Según datos de IBOPE, salud, educación y seguridad pública eran las principales motivaciones para participar de las protestas.

Por su parte, Zibechi (2013, p. 28) refiere a la trayectoria de nuevos movimientos urbanos como resistencia anticapitalista hacia un modelo de extractivismo urbano. Relativizando la influencia de las redes sociales, destaca que lo novedoso de junio de 2013 fue la masificación de las demandas de colectivos organizados, preexistentes al estallido de la calle, que luchan por el derecho a la ciudad tales como, el MPL resistiendo el aumento del boleto y los Comité Popular da Copa nacidos para resistir los traslados forzosos que suponían la reorganización urbana para albergar cuatro megaeventos deportivos. A partir de esta lectura, en tanto el precio del autobús es considerado un mecanismo de control social, la tarifa cero garantizaría entonces, simbólicamente, el acceso a otros derechos sociales como la salud, la educación y la cultura. De modo que, si los molinetes representan la exclusión, no solo en transporte, sino en la vida en general, la gratuidad del pasaje de ómnibus logra articular las diversas demandas surgidas en junio de 2013.

En consecuencia, se puede sostener que el MPL está inserto en la tradición de luchas anti capitalistas e igualitarias y ello permite encontrar elementos de lo que constituye un movimiento social en sentido fuerte. En esta instancia de la acción colectiva, el movimiento original rebasado por la ciudadanía se convirtió, en consonancia con un contexto de manifestaciones a nivel global, en una nueva modalidad de movimientos sociales que ha recibido el nombre de movimiento de indignados.

Hacia marzo de 2016:

Buena parte del poder de los movimientos deriva del hecho de que activan a gente sobre la que no tienen control, sin embargo, al irse ampliando el ciclo de protesta 
también se crean oportunidades para las élites y los grupos de oposición y los movimientos que iniciaron el ciclo pueden acabar teniendo cada vez menor influencia (Tarrow, 2004).

El abordaje de la evolución de las protestas de junio planteado por Singer (2013, p. 25) destaca el auge de las manifestaciones en la segunda fase, entre el 17 y 20 de junio, con la aparición de un número de demandas cada vez más vagas y diversas. Bringel y Pleyers (2015, p. 6) refieren a una indignación difusa ante la ambivalencia de discursos, la heterogeneidad de las demandas y la ausencia mediación de terceros y actores tradicionales. El desborde de los actores iniciales, comenzaba a dar cuenta de un cambio en la composición de las protestas.

La cada vez mayor heterogeneidad de demandas y reclamos, fue acompañada de un fuerte tono antipartidario que cristalizó para el PT la pérdida del monopolio de las calles (Goldstein 2019, p. 52). Hacia el 20 de junio de 2013, la reivindicación en contra de la corrupción comenzó a ganar espacios desplazando a los reclamos por mejores servicios públicos. Obtenida la revocación del aumento del boleto, la manifestación convocada para su conmemoración fue tomada por una gran masa verde-amarilla (Breda 2016, p. 9), ante lo cual, portavoces del MPL extendieron un comunicado en el cual denunciaban que grupos ajenos a sus intenciones se habían infiltrado a las marchas y que en consecuencia ponían fin a las manifestaciones. Encuestas realizadas por IBOPE, demuestran que en 2016 no sólo aumenta el apoyo a las manifestaciones por parte de la población respecto de 2013, sino que también las motivaciones para participar de las mismas se concentran en contra del gobierno federal.

Breda (2016, p. 8) señala, que los medios de comunicación, a diferencia de los inicios de junio donde tenían una actitud contraria a las manifestaciones y exigían a la policía el fin a las mismas, comenzaron a dar un giro en su postura y pasaron no solo a apoyar las protestas sino también a convocarlas, luego de comprender que "eran un buen negocio en tanto afectaban la popularidad de Dilma Rousseff" (Goldstein 2019, p. 50) amplificando una serie de demandas adicionales dirigidas contra el gobierno.

Cambiando la naturaleza de los reclamos, entraron en escena nuevos movimientos juveniles como el MBL, VPR y Revoltados On line que apropiándose de estéticas, prácticas y repertorios tradicionalmente de izquierda (Goldstein y Rezende 2016, p. 12) pasaron a protagonizar la protesta social. Tales movimientos compartían una serie de características comunes, como su origen reciente en las redes sociales, su declaración apartidaria, su aproximación ideológica al liberalismo económico y de una forma más o menos radical su combate hacia la corrupción, llegando incluso a solicitar una intervención militar como fue el caso de Revoltados On Line. Estos grupos "usaron con 
eficacia el potencial convocante de las redes, en un primer momento Orkut, antecedente de Facebook, para difundir ideas liberales que no eran parte de los debates principales en el país" (Rocha Camila, citado por Goldstein 2019, p. 53).

Luego de la reelección de Dilma Rousseff en 2014, por un escaso margen de 3.2 puntos en segundo turno, cuestionada como fraudulenta por la oposición, en medio de los escándalos de corrupción, en torno a las denuncias de sobornos relacionadas con la Operación Lava Jato, y de la ruptura de promesas de campaña, mediante la aplicación de un fuerte ajuste fiscal, la polarización social se acentuó.

A tres meses de asumido su segundo mandato, Dilma Rousseff enfrentó la gran marcha contra la corrupción que tuvo lugar el 15 marzo de 2015 en San Pablo. Poco a poco, entre una y otra manifestación de las convocadas por los nuevos grupos de derecha, la propuesta del impeachment fue cobrando fuerza en las calles (Breda 2016, p. 11). Un año después, hacia el 13 de marzo de 2016, tiene lugar la concentración política más grande de la historia democrática de Brasil. Alrededor de 500.000 personas reclamaban lisa y llanamente por el impeachment a la mandataria, cuyo proceso legal ya estaba iniciado en la Cámara de Diputados. Con una aversión a las políticas de gasto público y a los planes sociales y una radical oposición al PT, estos actos, protagonizados en su mayoría por sectores sociales altos, blancos y de mediana o avanzada edad, representan un cambio significativo respecto de la composición de las grandes protestas de 2013 (Tible y Moraes 2015, p. 7).

Se sostiene, que en este segundo momento de la protesta, el reclamo ya no es por más Estado sino por el contrario, más mercado. Los reclamos ya no referían a un aumento de ciudadanía, entendida desde la pertenencia a un Estado que debe aumentar y mejorar la oferta de sus servicios públicos, sino que la indignación iba dirigida hacia el Estado mismo o, en todo caso, hacia un tipo de estado progresista, el cual se consideraba rebalsado de atribuciones que el mercado podría, por sí solo, brindar de forma más eficiente. El movimiento de indignados, había difuminado sus indignaciones iniciales hasta concentrarse en concreto contra la corrupción y a favor del impeachment a la mandataria. En esta instancia, se hacen presente movimientos sociales que revisten características de movimientos en un sentido débil, siguiendo la distinción aportada por Svampa (2017).

\section{CONCLUSIONES}

Se constituyó en el ciclo excepcional de protesta social que tuvo lugar en Brasil a partir del 2013 una paradoja intrínseca entre dos instancias de indignación referentes a los reclamos por más Estado y más mercado. 
La idea de plantear los momentos de la protesta en modo dicotómico y antagónico, refiere al punto de clarificar, en torno al binomio protesta - movimiento, que la nueva modalidad de movimientos sociales expresados como indignaciones ciudadanas que aparecen en un primer momento, revisten características de lo que se ha identificado teóricamente como movimientos en sentido fuerte. En un primer momento de la protesta, se constituyeron reclamos por la construcción de una ciudadanía y pertenencia plena al Estado que fueron viabilizados por un movimiento que en términos de Zibechi (2013) encarna la tradición de lucha anticapitalista e igualitaria de los movimientos sociales.

En un segundo momento, por el contrario, se hacen presentes indignaciones ciudadanas de otro tipo, enfocadas hacia una suerte de accountability social contra la corrupción y específicamente movilizadas a favor del impeachment. Ello implica un reclamo esporádico y espasmódico, en el sentido de que una vez concretado el juicio político, esta ola de manifestantes se retiró de escena. Esta instancia entonces, reviste características de movimientos en un sentido débil, abocado a la acción contenciosa para acceder a la concreción de ciertos resultados.

Entre el ciclo de protesta 2013-2016, Brasil se debatió en torno a dos sociedades, una cercana al liberalismo igualitario y otra al liberalismo libertario, y el territorio de disputa fue la calle. Los reclamos en las protestas partían, en principio, de demandas por una sociedad con un Estado interventor y en defensa de los más desventajados de la sociedad y derivaron luego, hacia demandas por una sociedad con un Estado mínimo que se rija por las leyes del mercado.

Según De Sousa Santos (2015, p. 28) las reivindicaciones populares a nivel mundial surgieron de la memoria de un estado social de derechos, de las ruinas de la socialdemocracia. Continuando este razonamiento, puede argumentarse que la ola de protesta social ha derivado de la experiencia progresista en Brasil. Dado que ésta supuso una ampliación de derechos sociales para una gran parte de la sociedad considerada excluida, se dio pie al surgimiento de nuevas modalidades de movimientos sociales que bregaban por una profundización constante de derechos y de acceso a la ciudad. En el marco de un contexto global de indignaciones, las protestas parecen prevalecer sobre los movimientos, sin embargo, este trabajo ha intentado reflexionar a partir del binomio protesta - movimiento, acerca de la presencia de movimientos sociales detrás de las manifestaciones que conformaron los dos momentos de indignaciones en Brasil en un modo paradójico.

Junio de 2013 tuvo una magnitud sorpresiva e indirectamente sentó las bases para que marzo de 2016 fuera más grande. Paradójicamente aquellos sectores tradicionalmente contrarios a la lógica participativa aprendieron que las calles pueden ser tomadas. 


\section{BIBLIOGRAFÍA}

Anderson, P. (2016) "Crisis en Brasil", en Gentilli. P. (coord) Golpe en Brasil. Genealogía de una farsa. Buenos Aires: Clacso/ Editorial Octubre, pp. 35-64.

Braga, R. (2013) "Sob a sombra do precariado", en Maricato E. [et al.] Cidades rebeldes: Passe Livre e as manifestações que tomaram as ruas do Brasil. São Paulo: Boitempo/ Carta Maior.

Breda, T. (2016) "Brasil: Crónica de un impeachment anunciado. Los colores de un país escindido", Nueva Sociedad, N²63, pp. 4-18.

Bringel, B. (2013) Le Brésil et la géopolitique de I'indignation. La Vie des idées. Disponible en: https://laviedesidees.fr/Le-Bresil-et-la-geopolitique-de-l.html. (Acceso en: 08/05/2019).

Bringel, B. y Pleyers, G. (2015) "Junho de 2013... dois anos depois. Polarização, impactos e reconfiguração do ativismo no Brasil”, Nueva Sociedad, N88, pp. 4-17.

Castells, M. (2012) "Redes de Indignación y Esperanza". Madrid: Alianza.

Castells, M. (2013) "Brasil: escuchar la calle", La Vanguardia, Barcelona. Disponible en: https://www.lavanguardia.com/opinion/articulos/20130629/54377068734/brasilescuchar-a-la-calle.html. (Acceso en: 03/01/2019).

Ceceña, A.E. (2002) "Rebeldías sociales y movimientos ciudadanos", en OSAL (Observatorio Social de América Latina), Buenos Aires, N6, pp. 11-16.

De Sousa Santos, B. (2015) "Las revueltas mundiales de indignación: su significado para la teoría y la práctica", en Rodríguez, J.L.E.. (ed.) Revueltas de indignación y otras conversas, Bolivia: Proyecto Alice, pp. 17-36.

Domingues, J.M. (2013) "Las movilizaciones de junio de 2013: ¿Explosión fugaz o novísima historia de Brasil?", OSAL (Observatorio Social de América Latina), №34, pp. 63-73.

Goldstein, A. (2019) Bolsonaro. La democracia de Brasil en peligro. Buenos Aires, Marea.

Goldstein, A. y Rezende, R. (2016) "Brasil: fin de los gobiernos del PT, presente de crisis y resistencias", Revista Política Latinoamericana, N², pp. 1-16.

Iglesias, E. (2008) "Política y protesta. Visiones comparadas de la acción colectiva", en Fernández, A. y Lesgart C. (comps.) La democracia en América Latina. Partidos políticos y movimientos sociales. Rosario: Homo Sapiens, pp. 149-170. 
Iglesias, E. (2012) "De la colonización de la sociedad civil a las tensiones entre partidos en el gobierno y movimientos sociales", Lua Nova, N87, pp. 113-137.

Pesquisa CNI-IBOPE. Avaliação do governo. Disponible en: http://www.ibope.com.br/ptbr/noticias/Documents/Pesquisa CNI IBOPE Avaliacao do Governo Dezembro2015 v3.pdf. (Acceso en: 06/05/2019).

Pesquisa CNI-IBOPE. Retratos da sociedade brasileira. Manifestações populares: 2013 e 2016. Disponible en: https://bucket-gw-cni-static-cmssi.s3.amazonaws.com/media/filer public/44/d7/44d72eec-d6a9-4ed5-87e2cf0fd6a905ab/retratosdasociedadebrasileira 31 manifestacoes.pdf. (Acceso en: 06/05/2019).

Pesquisa IBOPE Inteligência. 89\% dos manifestantes não se sentem representados por partidos. Disponible en: http://www.ibopeinteligencia.com/noticias-e-pesquisas/89dos-manifestantes-nao-se-sentem-representados-por-partidos/. (Acceso en: 06/05/2019).

Rocca Rivarola, D. (2016) "De las Jornadas de Junho al Impeachment sem Crime: protestas, militancia oficialista y crisis política en Brasil (2013-2016)". Disertación en VI Jornadas de Estudios Políticos. Los usos del Estado. Proyectos políticos en disputa en las democracias de América Latina. Universidad Nacional de General Sarmiento (UNGS).

Rosanvallon, P. (2015) La contrademocracia. La política en la era de la desconfianza. Buenos Aires: Manantial.

Seoane, J.; Taddei, E. y Algranati, C. (2006) "Las nuevas configuraciones de los movimientos populares en América Latina", en A. A. Boron y G. Lechini (comps.), Política y movimientos sociales en un mundo hegemónico. Lecciones desde África, Asia y América Latina. Buenos Aires: Clacso, pp. 227-250.

Singer, A. (2013) "Brasil, junho de 2013. Classes e ideologias cruzadas", Novos Estudos CEBRAP, 97, pp. 23-40.

Svampa, M. (2017) Del Cambio de época al fin de ciclo. Buenos Aires, Edhasa.

Tarrow, S. (2004) El poder en movimiento. Los movimientos sociales, la acción colectiva y la política. España, Alianza.

Tible, J. (2013) “¿Una nueva clase media en Brasil? El lulismo como fenómeno políticosocial", Nueva Sociedad, N²43, pp.4-17.

Tible, J.; Moraes, A. (2015) “¿Fin de fiesta en Brasil?”, Nueva Sociedad, № 259, pp. 4-14. 
Tilly, C. (2000) “Acción colectiva”, Apuntes de investigación CECYP, N 6, pp. 3-30.

Touraine, A. (1998) Los movimientos sociales. En la producción de la sociedad. México: Universidad Nacional de México.

Vommaro, P. (2015) Juventudes y políticas en la Argentina y en América Latina: tendencias, conflictos y desafíos. Buenos Aires: Grupo Editor Universitario.

Zibechi, R. (2003) "Los movimientos sociales latinoamericanos: tendencias y desafíos", OSAL (Observatorio Social de América Latina), N 9, pp. 185-188.

Zibechi, R. (2013) "Debajo y detrás de las grandes movilizaciones", OSAL (Observatorio Social de América Latina), N³4, pp. 15-35. 